\title{
Approach to Isolated Trapezoid Fractures
}

\author{
Michael Nammour, MD, ${ }^{1}$ Bhumit Desai, BS, ${ }^{2}$ Michael Warren, MD, ${ }^{1}$ Brian Godshaw, MD, ${ }^{1,2}$ Misty Suri, MD ${ }^{1,2}$ \\ ${ }^{1}$ Department of Orthopedics, Ochsner Clinic Foundation, New Orleans, LA ${ }^{2}$ The University of Queensland Faculty of Medicine, Ochsner \\ Clinical School, New Orleans, LA
}

\begin{abstract}
Background: The trapezoid is the least commonly fractured carpal bone, comprising $4 \%$ of all carpal fractures. To date, few articles have been published on isolated trapezoid fractures. Mechanisms of injury have typically been reported as an axial load, with or without forced wrist flexion/extension, that is transmitted from the second metacarpal indirectly to the trapezoid.

Case Reports: Two patients presenting with symptoms of nonspecific wrist pain after acute trauma were initially worked up with plain film x-rays. Physical examinations identified nonspecific wrist pain in both patients. Mechanisms of injury involved direct trauma and an axial force transmitted through the scaphoid region of an extended wrist in each patient. Plain $\mathrm{x}$-rays were negative for trapezoid fracture in both patients. Computed tomography and magnetic resonance imaging revealed the diagnoses. Conservative management consisted of splinting and immobilization, with full recovery reported at 2.5- and 3-month follow-up. Conclusion: Isolated fractures of the trapezoid require a high index of suspicion as they are rare, and localizing signs and symptoms are typically vague and may mimic those of scaphoid fractures. When athletes present with dorsal wrist pain, swelling, and snuffbox tenderness in the setting of negative plain x-rays, the most likely mechanisms of injury are associated with athletic activity. Treatment depends on the degree of displacement and other associated injuries and ranges from activity modification or immobilization to open reduction with internal fixation.
\end{abstract}

Keywords: Carpal bones, fractures-bone, trapezoid bone, wrist joint

Address correspondence to Misty Suri, MD, Department of Orthopedics, Ochsner Clinic Foundation, 2700 Napoleon Ave., New Orleans, LA 70115. Tel: (504) 899-9311. Email: msuri@ochsner.org

\section{INTRODUCTION}

The trapezoid is the least commonly fractured carpal bone, comprising $4 \%$ of all carpal fractures. ${ }^{1,2}$ Isolated fractures of the trapezoid require a high index of suspicion as they are rare, and localizing signs and symptoms are typically vague and may mimic those of scaphoid fractures. ${ }^{3,4}$ In addition to this unimpressive clinical presentation, identifying fractures on plain radiographs can be difficult, and imaging with computed tomography (CT) or magnetic resonance imaging (MRI) is often necessary to achieve the diagnosis. Treatment depends on the degree of displacement and other associated injuries. Treatment options include activity modification, immobilization, and open reduction with internal fixation. . $^{3,5-7}$

We present the cases of 2 patients with isolated trapezoid fractures and provide an overview of the workup and management of trapezoid fractures.

\section{CASE REPORTS \\ Patient 1}

A 27-year-old female presented to the emergency department (ED) with right wrist pain following a high-velocity motor vehicle accident. The patient explained that her airbags deployed, and she experienced immediate 8/10 pain. Physical examination identified significant edema in the right wrist and significant tenderness to palpation over the radial aspect of the carpal bones. The patient's right wrist and hand were neurovascularly intact. Wrist $\mathrm{x}$-rays demonstrated a linear lucency within the trapezoid (Figure 1). Multiview hand x-rays showed no evidence of a displaced fracture or dislocations. Noncontrast CT of the hand confirmed an obliquely oriented nondisplaced fracture of the trapezoid bone extending to the articular surface of the carpometacarpal joint at the base of the second metacarpal (Figure 2). The ED physician made the diagnosis on initial assessment with the assistance of wrist plain films. The patient was discharged in a wrist splint with hand clinic follow-up in 2 days.

In clinic, management involved nonoperative care with a wrist splint for 1 week awaiting resolution of edema, followed by a transition to a thumb spica and radial gutter fiberglass cast in the intrinsic plus position for 4 weeks. After cast removal, the patient was transitioned to a thumb spica brace and prescribed 6 weeks of physical therapy and occupational therapy. At 10-week follow-up, the patient reported a full return to baseline function.

\section{Patient 2}

A 23-year-old male presented to the outpatient sports medicine clinic with right wrist pain following an injury at baseball practice. The patient was participating in a dynamic 


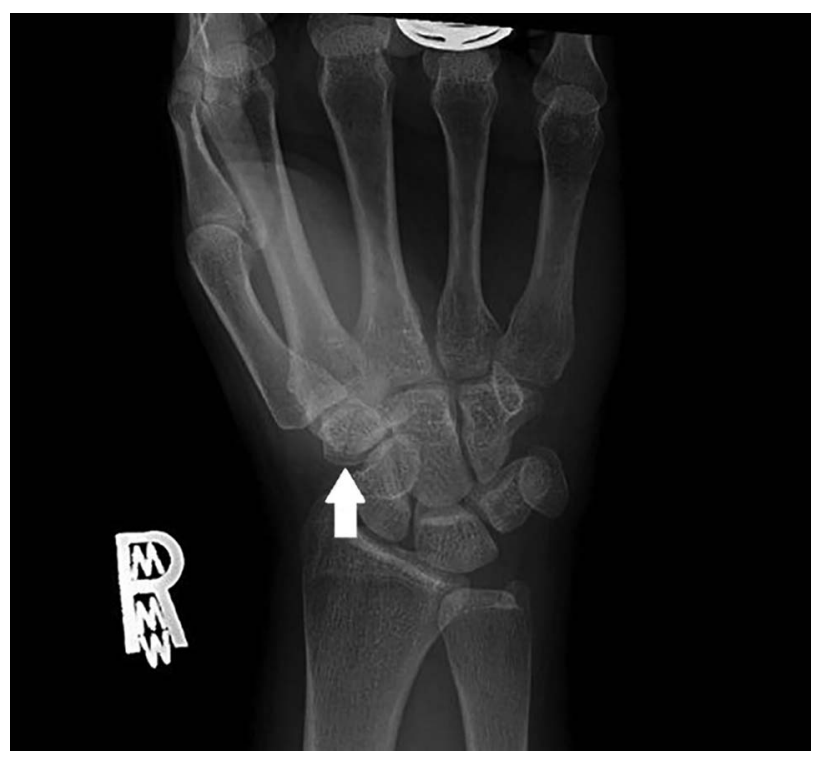

Figure 1. Patient 1: Posterior-anterior wrist x-ray shows a linear lucency in the right trapezoid (arrow).

exercise movement in which he moved from a standing position down into a push-up position with his wrists absorbing the majority of the force. The mechanism of injury involved an axial force transmitted through the scaphoid region of an extended wrist to the trapezoid. His clinical presentation was significant for constant dull pain at the anatomic snuffbox with throwing motion and tenderness to palpation at the scaphoid. The patient had no edema or limitation in range of motion, he was neurovascularly intact, and his range of motion was within normal limits. All special tests of the hand and wrist-Phalen, Durkan, Tinel, Finkelstein, piano key, Watson scaphoid shift, Allen, Froment sign, carpometacarpal grind, and Bunnell-were unremarkable. At initial assessment, hand $\mathrm{x}$-rays showed no acute bony abnormalities (Figure 3). MRI of the right wrist, ordered to rule out scaphoid fracture, showed a nondisplaced fracture of the dorsal one-third of the trapezoid with moderate associated marrow edema (Figure 4). CT of the right wrist confirmed a stable nondisplaced fracture of the dorsal aspect of the trapezoid. Time to diagnosis was 1 day after initial assessment and 8 days after the injury. The patient was managed nonoperatively with a right wrist cast for 6 weeks followed by 6 weeks of physical therapy and occupational therapy. At 12-week follow-up, the patient reported an absence of pain and a return to baseline function of the right hand and wrist.

\section{DISCUSSION}

To date, very few articles have been published in English on isolated trapezoid fracture; information is derived exclusively from case reports. ${ }^{3,6,8-17}$ (We found 5 non-English reports of isolated trapezoid fracture. ${ }^{18-22}$ ) The cause for the infrequency of this diagnosis is yet to be determined, but possible underlying reasons include underdiagnosis by providers or low incidence because of strict anatomic positioning. The anatomic shape and position of the trapezoid make it highly stable and difficult to fracture. ${ }^{14}$ The trapezoid lies in the distal carpal row and has very

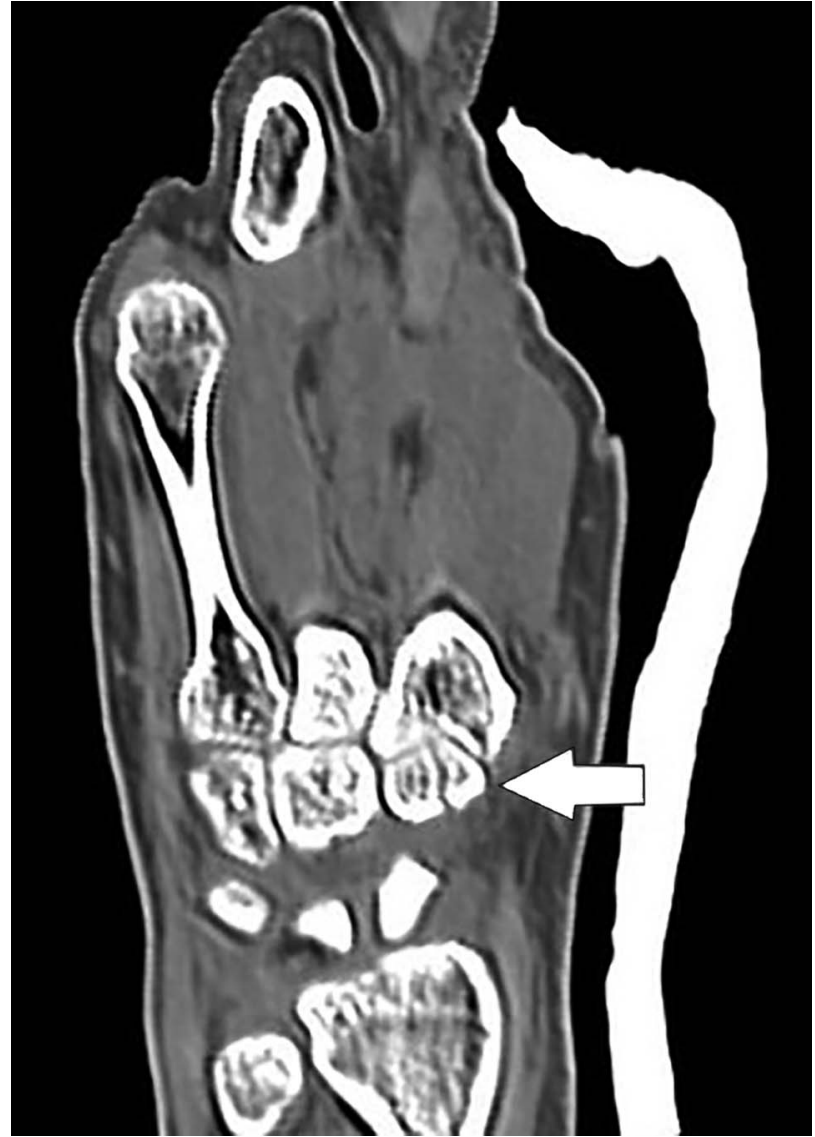

Figure 2. Patient 1: Noncontrast coronal computed tomography shows a nondisplaced oblique right trapezoid fracture (arrow).

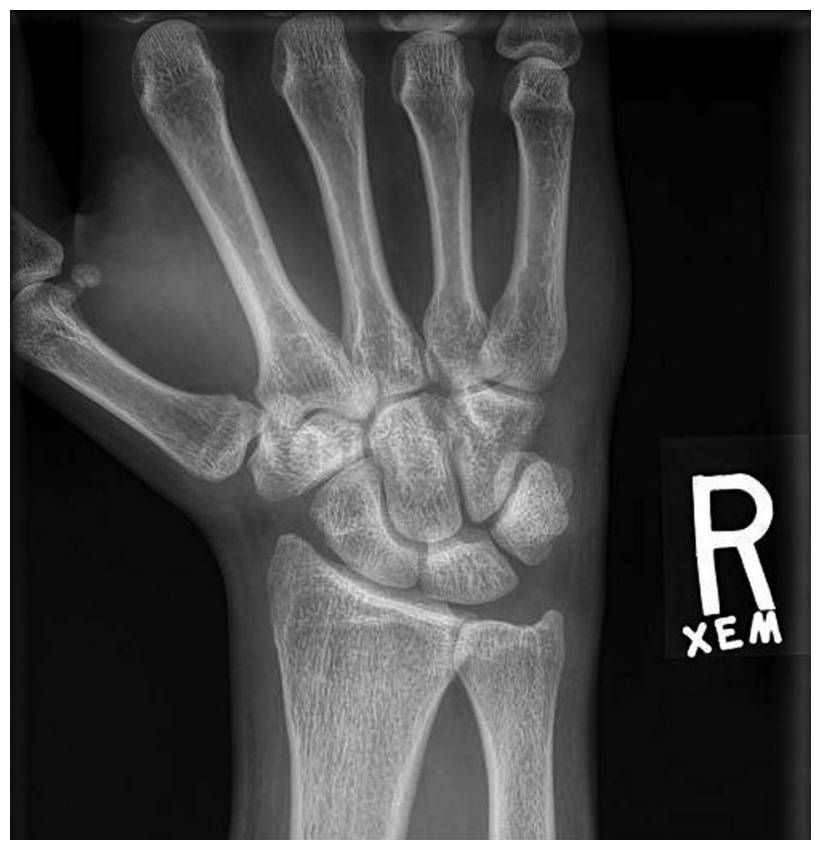

Figure 3. Patient 2: Posterior-anterior wrist x-ray shows no evidence of acute fracture. 


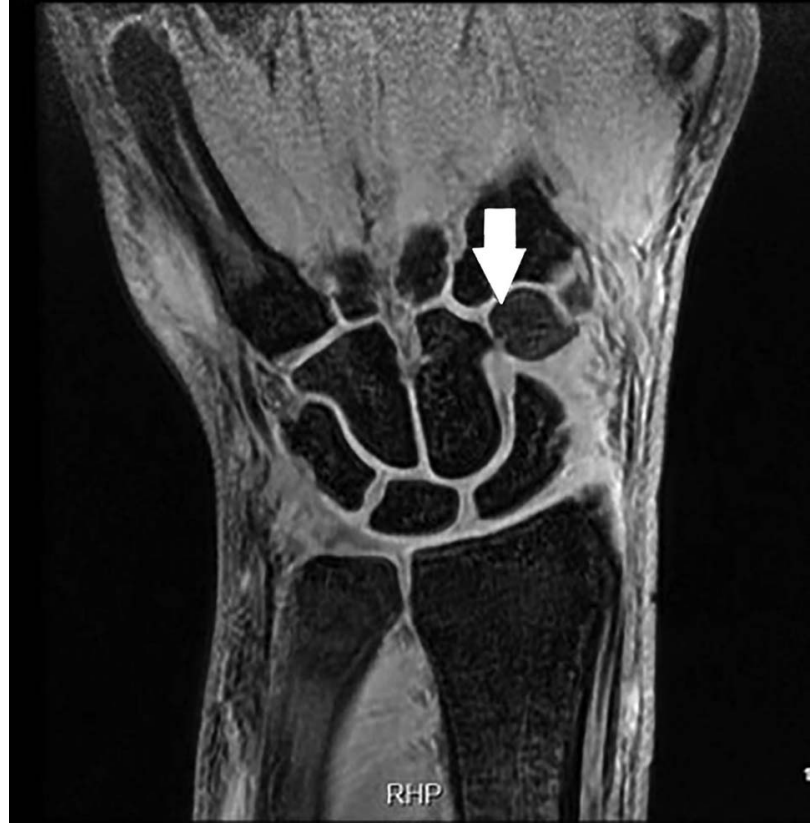

Figure 4. Patient 2: Noncontrast coronal magnetic resonance imaging shows nondisplaced fracture of the dorsal one-third of the right trapezoid (arrow).

strong ligamentous attachments to the second metacarpal base distally, the trapezium radially, the capitate ulnarly, and the scaphoid proximally. ${ }^{23}$ These strong ligamentous attachments make the trapezoid relatively immobile. These attachments, combined with the keystone shape of the trapezoid, make it twice as wide dorsally than volarly. A considerable amount of trauma, usually involving axial loading with forced wrist flexion or extension, is therefore required to cause injury. ${ }^{11}$ The mechanism of injury has typically been reported as an axial load, with or without forced wrist flexion/extension, transmitted from the second metacarpal indirectly to the trapezoid. ${ }^{1,17,24,25}$ The axial force is transmitted from the second metacarpal base through the trapezoid to the scaphoid. ${ }^{16,23}$ In instances of metacarpal flexion or a volarly directed force, an axial load can cause a dorsal shear fracture of the trapezoid with concomitant dorsal subluxation of the second metacarpal at the carpometacarpal joint. ${ }^{8,10}$ Another frequently reported cause is direct high-energy trauma that often results in fractures involving the surrounding carpal bones and metacarpals..$^{9,12,14}$

Isolated trapezoid fractures are extremely rare, and to date, only 7 cases (including Patient 2 in this article) of isolated trapezoid injuries directly related to sports injuries have been reported. ${ }^{8,13,16}$ In a 3-year retrospective study, Blomquist et al reviewed 64 confirmed trapezoid fractures and found that only 3 patients had isolated trapezoid fractures; all 3 were related to sports injuries in Division $1 \mathrm{~A}$ collegiate athletes. ${ }^{8}$ In all 3 patients, the fracture was not identified on plain $\mathrm{x}$-ray, and CT scan was negative for fracture in 2 of the 3 patients. MR arthrogram identified marrow edema and coronal-oriented fractures in all 3 patients. The first patient, a tennis player with a nondisplaced coronal fracture, was treated with 6 weeks of activity modification (time off from tennis) and splint immobilization. Her wrist pain resolved in 1 month, and she was playing competitive tennis 2 months after treatment initiation. The second patient, a soccer goalie with a nondisplaced coronal fracture, was initially treated with a thumb spica splint, physiotherapy, and an ultrasound bone stimulator while she continued to play. However, follow-up MRI 4 months later showed progression of the fracture, and the patient subsequently was treated with 8 weeks of full-time rest and immobilization that resulted in complete pain resolution and fracture healing. The third patient, a middle linebacker with a coronal-oriented $1 \mathrm{~mm}$ displaced fracture, was initially treated with a highly protective club cast because he desired to continue playing. One month later, the patient elected to undergo surgery as he had significant discomfort that prevented him from competing at the level he desired in addition to a lack of radiographic healing. Surgery revealed that a portion of the dorsal wrist capsule had become interposed proximally into the fracture, possibly preventing healing. The fracture was stabilized with screw fixation, and although the patient did not return to play that season, he subsequently played professional football. ${ }^{8}$

Isolated trapezoid fractures are even rarer when the mechanism involves trauma because the other carpal bones are almost always involved. ${ }^{7}$ One reported case of traumatic isolated trapezoid fracture involved an excessive flexion stress indirectly placed on the trapezoid through the second metacarpal. ${ }^{14}$ Although the trapezoid fractures in our report occurred in different contexts, the patients shared a similar mechanism of injury. Patient 1 was holding a steering wheel, placing her wrist in forced extension. When her vehicle impacted at a high velocity, the trauma was transmitted through the steering wheel to the scaphoid region. A subsequent axial force through the scaphoid to the trapezoid may explain her radiographic and clinical findings. Because of the rigid fixation of the trapezoid, the stress applied has been likened to the manner in which a walnut is broken with a nutcracker. ${ }^{14}$ In terms of fracture healing, the extensor carpi radialis longus (ECRL) and the extensor carpi ulnaris (ECU) may also be deforming forces. ${ }^{7}$ The dorsal attachments of the ECRL and ECU tendons subject the trapezoid bone to a traction force that may contribute to delayed osseous union.

Patients with trapezoid fractures may complain of point tenderness at the base of the second metacarpal, pain with range of motion at the second metacarpophalangeal joint, or pain and tenderness at the anatomic snuffbox. Patients may or may not have dorsal wrist swelling and decreased range of motion at the wrist. The presence of dorsal wrist swelling and decreased range of motion has been postulated to be the result of a dorsally displaced fracture fragment. ${ }^{8}$ The concern for underdiagnosis may be attributed to the efficacy of imaging used to diagnose carpal bone fractures in patients who present with hand pain. Localizing signs and symptoms vary and may mimic scaphoid fracture..$^{3,4}$

Previous case studies exhibit that plain films can be misleading, and CT or MRI is often necessary for confirmatory diagnosis. Diagnosis may be delayed if no further imaging is performed. Kain and Heras-Palou conducted a retrospective review of all trapezoid fractures from 2001-2011 at their institution. ${ }^{16}$ They reviewed case notes on mechanism of injury, fracture pattern, mode of diagnosis, and time to 
diagnosis and treatment. Of the 11 patients eligible for review, only 7 patients had fractures consistent with the diagnosis of trapezoid fracture. ${ }^{16}$ The fracture patterns included sagittal (6), coronal (4), and crush (1). All but 1 of the 11 patients underwent further imaging with $\mathrm{CT}, \mathrm{MRI}$, or both. None of the coronal fractures could be seen on plain films and required $\mathrm{CT}$ or $\mathrm{MRI}$ for diagnosis, whereas the sagittal and crush injuries were successfully detected on plain $\mathrm{x}$-ray. Five of the 11 trapezoid fractures were found to be in isolation, but the authors did not state which fracture patterns were associated with isolated injury. Only 2 of the 11 fractures were displaced enough to require open reduction and internal fixation. With their review, Kain and Heras-Palou highlight the importance of further imaging when a trapezoid fracture is suspected, as plain x-ray is not sufficient to rule out coronal fracture patterns. ${ }^{16}$

The efficacy of radiographs can be better understood by classifying isolated trapezoid fractures as nondisplaced or displaced fractures. Displaced fractures are often associated with adjacent metacarpal or carpal bone fractures and are therefore readily identifiable on plain films. ${ }^{7,26}$ Unlike with scaphoid fractures, no evidence in the literature suggests that radiographic resorption becomes more apparent over time. However, in practice environments where advanced imaging is unavailable, occult trapezoid fractures can be managed with serial radiographs and immobilization. In a case of a nondisplaced isolated trapezoid fracture in which radiographs were initially not diagnostic, immobilization with a short arm thumb splint is an appropriate choice for initial management. ${ }^{3}$ Persistent pain at follow-up has been used as an indication for advanced imaging that eventually leads to a confirmatory diagnosis. ${ }^{12}$

Although technetium bone scans have been used to diagnose occult trapezoid fractures, CT and MRI are more readily available, take less time, and provide better detail for treatment planning. ${ }^{17}$ The choice of CT or MRI as the ideal imaging modality is a subject of debate. Kain and Heras-Palou recommend $\mathrm{CT}$ as the radiologic investigation of choice; however, Blomquist et al found that MRI was more sensitive and specific for diagnosing isolated trapezoid fractures. 8,16 Blomquist et al also found that MRI provided more information about the absence of secondary injury findings and was more helpful for treatment plan formulation. ${ }^{8}$

Generalizations about management of isolated trapezoid fractures are principally based on the few case reports available in English and on individual surgeon experience. Isolated trapezoid fractures that are nondisplaced or minimally displaced appear to have uneventful union and good functional results when treated with activity modification and immobilization. 3,5-7,12,14 Fractures that are displaced or associated with the second metacarpal or other carpal fractures have been treated successfully with operative fixation through a dorsal approach. ${ }^{7,9,13}$ Even when surgical fixation was performed several months after injury, patients achieved union with good functional outcomes. ${ }^{7,13}$ Dorsal avulsion fractures may hinge distally and allow capsular interposition between the fracture fragments proximally, which may prevent healing. ${ }^{8}$

Failure to identify and treat trapezoid fractures can cause long-term complications including nonunion, malunion, avascular necrosis, pain, and diminished grasp strength. ${ }^{7,13,15}$

\section{CONCLUSION}

Isolated trapezoid fractures are extremely rare and require a high degree of clinical suspicion. Athletes with dorsal wrist pain, swelling, and snuffbox tenderness in the setting of negative plain $x$-rays require particular attention. With the increase in use of CT and MRI, isolated trapezoid fractures may be more commonly diagnosed in the future.

\section{ACKNOWLEDGMENTS}

The authors have no financial or proprietary interest in the subject matter of this article.

\section{REFERENCES}

1. Garcia-Elias M. Carpal bone fractures (excluding scaphoid fractures). In: Watson K, Weinzweig J, eds. The Wrist. Philadelphia, PA: Lippincott Williams \& Wilkins; 2001:173-186.

2. Papp S. Carpal bone fractures. Orthop Clin North Am. 2007 Apr;38(2):251-260, vii. doi: 10.1016/j.ocl.2007.03.003.

3. Jeong GK, Kram D, Lester B. Isolated fracture of the trapezoid. Am J Orthop (Belle Mead NJ). 2001 Mar;30(3):228-230.

4. Waizenegger M, Wastie ML, Barton NJ, Davis TR. Scintigraphy in the evaluation of the "clinical" scaphoid fracture. J Hand Surg Br. 1994 Dec;19(6):750-753.

5. Green DP, Pederson WC. Green's Operative Hand Surgery. 5th ed. Philadelphia, PA: Elsevier/Churchill Livingstone; 2005.

6. Yasuwaki Y, Nagata Y, Yamamoto T, Nakano A, Kikuchi H, Tanaka S. Fracture of the trapezoid bone: a case report. $J$ Hand Surg Am. 1994 May;19(3):457-459. doi: 10.1016/0363-5023(94)90062-0.

7. Pruzansky M, Arnold L. Delayed union of fractures of the trapezoid and body of the hamate. Orthop Rev. 1987 Sep;16(9):624-628.

8. Blomquist GA, Hunt III TR, Lopez-Ben RR. Isolated fractures of the trapezoid as a sports injury. Skeletal Radiol. 2013 May;42(5):735-739. doi: 10.1007/s00256-013-1581-z.

9. Watanabe $\mathrm{H}$, Hamada $Y$, Yamamoto $Y$. A case of old trapezoid fracture. Arch Orthop Trauma Surg. 1999;1 19(5-6):356-357.

10. Ting MH, Tompson JD, Ek ET. Isolated dislocation of the trapezoid. Hand Surg. 2012;17(3):391-393. doi: 10.1142/S0218810412720392.

11. Sadowski RM, Montilla RD. Rare isolated trapezoid fracture: a case report. Hand (N Y). 2008 Dec;3(4):372-374. doi: 10.1007/s11552-008-9100-8.

12. Nijs $\mathrm{S}$, Mulier T, Broos P. Occult fracture of the trapezoid bone: a report on two cases. Acta Orthop Belg. 2004 Apr;70(2):177-179.

13. Nagumo A, Toh S, Tsubo K, Ishibashi Y, Sasaki T. An occult fracture of the trapezoid bone. A case report. J Bone Joint Surg Am. 2002 Jun;84-A(6):1025-1027.

14. Miyawaki T, Kobayashi M, Matsuura S, Yanagawa H, Imai T, Kurihara K. Trapezoid bone fracture. Ann Plast Surg. 2000 Apr;44(4):444-446.

15. Kam ML, Sreedharan S, Teoh LC, Chew WY. Severe isolated trapezoid fracture: a case report. Hand Surg. 2011;16(2):185-187. doi: 10.1142/S0218810411005321.

16. Kain N, Heras-Palou C. Trapezoid fractures: report of 11 cases. J Hand Surg Am. 2012 Jun;37(6):1159-1162. doi: 10.1016/j.jhsa.2012.02.046.

17. Gruson KI, Kaplan KM, Paksima N. Isolated trapezoid fractures: a case report with compilation of the literature. Bull NYU Hosp Jt Dis. 2008;66(1):57-60.

18. Kuhlmann JN, Fournol S, Mimoun M, Baux S. Fracture of the trapezoid bone. Apropos of a case report [in French]. Ann Chir Main. 1986;5(2):133-134. 
19. Jensen BV. Fracture of the trapezoid bone [in Danish]. Ugeskr Laeger. 1990 Mar 5;152(10):676-677.

20. Jacoulet $P$, Lautman $S$, Mraovic T. Isolated trapezoid fracture: a case report [in French]. Chir Main. 2009 Dec;28(6):378-380. doi: 10.1016/j.main.2009.08.011.

21. Filloux JF, Morfaux V, Jarde O, Vives P. A defective callus of the trapezoid bone diagnosed by 3-dimensional scanner [in French]. Acta Orthop Belg. 1996 Sep;62(3):180-182.

22. De Simone M. Isolated fracture of the trapezoid bone [in Italian]. G Med Mil. 1954 Jan-Feb;104(1):77-79.
23. Cohen MS. Fractures of the carpal bones. Hand Clin. 1997 Jan-Feb;13(4):587-599.

24. Volz RG, Lieb M, Benjamin J. Biomechanics of the wrist. Clin Orthop Relat Res. 1980 Jun;(149):112-117.

25. Stein AH Jr. Dorsal dislocation of the lesser multangular bone. J Bone Joint Surg Am. 1971 Mar;53(2):377-379.

26. Calfee RP, White L, Patel A, Stern PJ. Palmar dislocation of the trapezoid with coronal shearing fracture: case report. J Hand Surg Am. 2008 Nov;33(9):1482-1485. doi: 10.1016/j.jhsa.2008.06.009.

This article meets the Accreditation Council for Graduate Medical Education and the American Board of Medical Specialties Maintenance of Certification competencies for Patient Care and Medical Knowledge. 